\title{
El primer punto de cambio del gobierno cubano: III Congreso del Partido Comunista Cubano de 1986*
}

The First Point of Change in the Cuban Government: 3rd Congress of the Cuban Communist Party in 1986

Carlos David Carrasco Muro ${ }^{\text {a }}$

Universidad Católica Andrés Bello, Venezuela

carlosdavid1993@gmail.com

ORCID: http://orcid.org/0000-0002-2837-5926
DOI: https://doi.org/10.11144/Javeriana.papo24-1.ppcg Redalyc: http://www.redalyc.org/articulo.oa?id=77760192003

Fecha de recepción: 05 Septiembre 2018 Fecha de aprobación: 10 Diciembre 2018

Fecha de publicación: 24 Junio 2019

\section{Resumen:}

Este trabajo se trata de un ensayo histórico sobre el III Congreso del Partido Comunista Cubano de 1986, sobre la base de un análisis del contexto internacional y los discursos pronunciados por Fidel y Raúl Castro en las plenarias de la reunión partidista. La justificación de este trabajo se basa en la necesidad de entender la naturaleza de los cambios políticos del gobierno cubano en los últimos años de la Guerra Fría, marcados por la precariedad económica y las transiciones geopolíticas en América Latina.

Palabras clave: Cuba, Castro, comunismo, Guerra Fría, América Latina.

\section{Abstract:}

This paper is a historic essay about the 3rd Congress of the Cuban Communist Party in 1986 developed as an analysis of the international context and the discourses by Fidel Castro and Raúl Castro in the party plenary sessions. This work is justified on the basis of a need to understand the nature of the political changes by the Cuban government in the latest years of the Cold War. This era was marked by the economic precariousness and the geopolitical transitions in Latin America.

Keywords: Cuba, Castro, Communism, Cold War, Latin America.

\section{Introducción}

En una época marcada por los acuerdos de paz en Colombia y una aguda crisis humanitaria en Venezuela, cuya influencia cubana en ambos casos es fundamental, resulta necesario un análisis político e histórico que permita apreciar el comportamiento del gobierno cubano ante los cambios graduales de la geopolítica. Pero este análisis no se puede realizar desde la inmediatez; la política cubana al igual que su economía subyace en una planificación y anticipación con actitud pragmática.

Por esta razón, resulta necesario estudiar el proceso político experimentado por el régimen cubano en 1986, con la celebración del III Congreso del Partido Comunista Cubano (PCC). Este evento, según los registros de voceros de la época, fue un punto de inflexión por las consideraciones y rectificaciones respecto a las acciones de la Revolución Cubana, luego de 27 años de gobierno y 11 años del primer congreso del PCC.

Desde un punto de vista histórico un poco más amplio, este hecho resulta muy peculiar para el estudio, ya que las acciones de este congreso se desarrollaron bajo un contexto internacional que cambiaría drásticamente el statu quo de la época.

La premisa o hipótesis desarrollada se centra en indicar que los cambios políticos de Cuba en 1986 fueron una reacción anticipada ante las decisiones tomadas a nivel mundial bajo el esquema de la Guerra de la Fría y sus implicaciones en la región de América Latina. Por esa razón, antes de analizar con mayor grado de

Notas de autor:

\footnotetext{
a Autor de correspondencia. Correo electrónico: carlosdavid1993@gmail.com
} 
profundidad el III congreso del PPC, se mencionan algunos hechos dentro del escenario político global y cubano, que permiten contextualizar la posición de Cuba durante la década de 1980, previa al congreso.

Para este ensayo histórico existieron limitaciones respecto al acceso a fuentes que permitieran una visión integral sobre este hecho histórico. El hermetismo cubano dificulta el acceso a una información de mayor grado de exactitud. No obstante, se pudo analizar a partir de discursos emitidos por Fidel y Raúl Castro; así como documentos oficiales y de libre acceso del PPC en Internet.

La motivación para realizar este estudio preliminar se basa en una inquietud académica por conocer más sobre el proceso interno de la política cubana, especialmente luego de la llegada de Fidel Castro al poder. Resulta necesario poder trascender las visiones simplistas e ideológicas, que impiden abordar en su justa dimensión a un país cuya verdadera influencia y grado de complejidad se desconoce, particularmente entre los jóvenes latinoamericanos de hoy.

También al vivir en un contexto como el venezolano, existe un amplio interés por conocer más sobre la historia de Cuba y sus implicaciones en Latinoamérica, sobre todo por el papel que juega dentro de las acciones políticas de Venezuela, desde la llegada al poder de la Revolución Bolivariana de Hugo Chávez en 1998.

\section{Antesala a la rectificación}

La división de la historia por etapas, inclusive por años, solo es una interpretación parcial y deliberada de los hechos del pasado. No obstante, esto permite analizar con mayor detalle ciertos momentos y acontecimientos, que ante la magnitud de las facetas de la civilización humana se pueden pasar por alto.

La antesala a la rectificación se inicia en la década de 1980, una época marcada por la transformación del panorama mundial. El sistema internacional empieza a mostrar signos de agotamiento ante la presión cada vez mayor del hemisferio occidental en contra de la Unión Soviética por su falta de libertades y armamento nuclear. En enero de 1980, los Estados Unidos de América y la Comunidad Europea decretan un embargo de cereales hacia la URSS como medida de presión (Carter, 1980, 7 de enero), y ya se asoma la posibilidad de un boicot contra los Juegos Olímpicos de Moscú de ese año. Todo esto, bajo el telón de fondo de una profunda tensión en Afganistán, la estabilización de la Revolución Islámica en Irán, la consolidación de la Revolución Sandinista en Nicaragua, y la elección del republicano Ronald Wilson Reagan como presidente de EE. UU frente a James Carter.

La Cuba de Fidel Castro no escapa a esta tendencia mundial de cambios. Para abril de 1980, inicia un movimiento multitudinario de solicitud de asilo político en la embajada de Perú en la Habana, que finaliza en el conocido episodio del éxodo del Mariel, donde aproximadamente 125 mil cubanos salieron de la isla con destino a Estados Unidos ${ }^{[1]}$. De igual forma, económicamente Cuba se encuentra con una economía en descrecimiento. Según el Banco Mundial, para ese año se mostraba un descenso de $-4,849$ \% del Producto Interno Bruto (PIB) (Banco Mundial, s. f.). En diciembre se realiza el Segundo Congreso del Partido Comunista de Cuba, en el Palacio de las Convenciones de Ciudad de La Habana, donde se expuso un balance del período entre 1975 y 1980, que destacaba la importancia de la relación vital que mantenía el gobierno cubano con la Unión Soviética.

Para el año de 1981, la tendencia de las revoluciones a nivel mundial continuaba permeando a las naciones, especialmente en Centroamérica: en El Salvador, se inicia una ofensiva contra el Estado por parte del Frente Farabundo Martí para la Liberación Nacional ${ }^{[2]}$. El Ejército Guerrillero de los Pobres de Guatemala [3] también protagoniza varios hechos subversivos, y un pequeño grupo guerrillero llamado "La Familia" planifica acciones violentas en Costa Rica con poco éxito. Por otro lado, la sombra de los atentados magnicidas se yergue sobre el mundo: el 13 de mayo de 1981, Mehmet Ali Ağca dispara contra el papa Juan Pablo II en la Plaza de San Pedro de El Vaticano, conmocionando a la Iglesia Católica y sus feligreses. El clima de guerra también adquiere otra dimensión con el inicio del conflicto armado entre Irán e Irak. 
Mientras esto pasa a nivel internacional, en Cuba se manifiesta un amplio interés por elevar su perfil como actor mundial. Durante 1981, se realiza el II Congreso de Economistas del Tercer Mundo en La Habana, donde se plantea la necesidad de un nuevo orden económico. Por su parte, Fidel Castro visita la URSS para participar en el XXVI Congreso del Partido Comunista Soviético, un hecho simbólico ampliamente difundido en aquel momento, enmarcado en una escalada de tensión entre las superpotencias por la política de Reagan. La isla caribeña también es sede ese año del encuentro de la Presidencia del Consejo Mundial de la Paz. En este evento se evidencia la preocupación de Cuba por la finalización de la etapa de distención entre EE. UU y la URSS. Fidel Castro (1981) deja entrever esto desde la perspectiva económica, denunciando el gasto militar de Estados Unidos: "La nueva administración plantea aumentos sostenidos en estos gastos en los próximos años de tal forma que en 1985 podría llegarse a la increíble asignación de 300 o 330.000 millones de dólares para fines guerreristas" (Castro, 1981).

Con la llegada del año 1982, el contexto bélico eleva su perfil simbólico y real. Ese año, la Organización del Tratado de Atlántico Norte (Otan) realiza sus primeras maniobras navales en el Mar Caribe y en el Golfo de México. En Suramérica se produce un conflicto armado entre Argentina y Reino Unido, conocido como la Guerra de las Malvinas o Falklands War ${ }^{[4]}$. Este conflicto armado genera profunda preocupación en la región de América Latina. También las denuncias por genocidio de organizaciones sociales hacia el gobierno de Guatemala se elevan en la opinión pública internacional. En el medio de la tensión bélica y los enfrentamientos internos de Centroamérica, Ronald Reagan realiza su primera gira por el conteniente americano. Sin embargo, uno de los hechos de mayor relevancia ocurre con el fallecimiento del soviético Leonid Brézhnev. Esto marca un nuevo proceso interno en la Unión Soviética, en búsqueda de un nuevo liderazgo. El miembro del buró político soviético Yuri Andrópov tomará las riendas de la superpotencia comunista.

Ante un año con la tensión en su máxima escala, no debe extrañar que Cuba haya tenido una agenda igualmente movida en lo referente a su política interna e internacional. En 1982, la isla celebra el X Congreso Mundial Sindical. Ante este auditorio mundial, estratégicamente importante para el gobierno cubano, Fidel Castro señala en su discurso las principales preocupaciones desde su perspectiva geopolítica, incluyendo las crisis en Sudáfrica, Namibia y Angola, así como las guerras civiles en El Salvador, Honduras y Guatemala. Igualmente, menciona los contingentes internacionalistas cubanos de Angola y Etiopía en un intento por visibilizar el poder bélico que tiene la isla comunista. Bajo el panorama de tensión en América Latina, en el marco del XVI Aniversario del desembarco del Granma y de la fundación de las Fuerzas Armadas Revolucionarias, Castro critica la gira de Reagan por la región y la posición que mantuvo frente al conflicto en el sur del Atlántico, entre Argentina y Reino Unido. De igual forma, el líder cubano aprovecha ese suceso para deslegitimar la Organización de los Estados Americanos y el Tratado Interamericano de Asistencia Recíproca (Tiar), posicionando al grupo de Países No Alineados ${ }^{[5]}$ como opción estratégica.

En 1983, la región de Centroamérica y el Caribe es protagonista dentro del contexto internacional. Esto se evidencia en hechos bélicos y simbólicos transcendentales. En marzo, el papa Juan Pablo II recorre varios países de América Central. En abril asesinan en Managua, Nicaragua, a la guerrillera salvadoreña Ana María, fundadora del Frente Farabundo Martí para la Liberación Nacional. En Guatemala, el general Óscar Humberto Mejía Víctores ejecuta un golpe de Estado derrocando al dictador Efraín Ríos Montt ${ }^{[6]}$. Para julio, patrulleros de Honduras y Nicaragua entablan un breve combate naval, y buques de guerra estadounidenses navegan frente a las costas de Nicaragua. Por su parte, Ronald Reagan se posiciona con diversas acciones a nivel estratégico, ya que anuncia el desarrollo del Sistema Estratégico de Defensa, popularmente conocido como "Star Wars" [7]. También gestiona la creación de la Fundación Nacional para la Democracia (NED) ${ }^{[8]}$, cuyo objetivo es ayudar a los grupos que están a favor de la democracia en el continente americano; y se realiza la invasión de Estados Unidos a la isla de Granada ${ }^{[9]}$ por 51 días, con el trasfondo de la Guerra Fría. 
En el convulsionado año de $1983^{[10]}$, en el contexto cubano se pueden apreciar los efectos de un panorama internacional complicado. Fidel Castro empieza a mostrar síntomas de una preocupación económica mayor. En el XXX aniversario del ataque al Cuartel Moncada, Castro señala la importancia de las resoluciones tomadas por el Comité Central del Partido para enfrentar las dificultades, ahorrar combustible y materias primas, así como elevar la eficiencia en la producción y los servicios. También señala las acciones militares que pretenden tomar ante las crisis evidenciadas durante ese año en el Caribe y América Central: "En fecha reciente, se llevó a cabo el ejercicio estratégico Bastión 83, con la participación de más de 100.000 personas, que incluía los principales cuadros del Partido, del Estado y de las Fuerzas Armadas Revolucionarias" (Castro 1983a). Por otro lado, en el discurso de despedida por los cubanos caídos en Granada, Castro señalaba que Cuba no tuvo responsabilidad en el golpe de Estado y la muerte del presidente Maurice Bishop, que no pensaba invadir y ocupar Granada, y que este país caribeño no se estaba convirtiendo en una base militar soviético-cubana (Castro, 1983b).

Para el año 1984, el contexto internacional, especialmente en América Latina, atraviesa por procesos de mayor distención. En enero, los representantes de Costa Rica, El Salvador, Guatemala, Honduras y Nicaragua, a través de la participación de los países del Grupo de Contadora ${ }^{[11]}$, firman un anteproyecto de paz para Centroamérica. Por otro lado, el Tribunal Internacional de La Haya sentencia que Estados Unidos debe suspender el bloqueo y minado de los puertos de Nicaragua. En este mismo país centroamericano, el FSLN gana las elecciones generales. No obstante, en el marco de la Guerra la Fría se sigue elevando la tensión. El fallecimiento del soviético Yuri Andrópov desencadena una nueva crisis en el liderazgo de la URSS. El miembro del buró político Konstantin Chernenko asume las riendas del bloque comunista. En Alemania Oriental, se despliegan misiles soviéticos SS-20. Mientras tanto, en Estados Unidos el presidente Ronald Reagan consigue su reelección, legitimando la política exterior que está aplicando.

Durante 1984 Fidel Castro inicia un nuevo ciclo en su relación con las potencias occidentales, acompañado del presidente de Nicaragua, Daniel Ortega. Por otro lado, en el ámbito militar, se quiere hacer una demostración de fuerza. Para el 26 de Julio de 1984, en el XV Congreso de la CTC ${ }^{[12]}$, efectuado en el teatro Lázaro Peña de La Habana, Castro convocó un segundo llamamiento de otro medio millón de hombres y mujeres para integrar las Milicias de Tropas Territoriales. También, el líder cubano muestra irrestricto apoyo a los movimientos revolucionarios de El Salvador y al gobierno nicaragüense. Sin embargo, ofrece una muestra inequívoca de querer un acercamiento con Occidente, ya que ese año, antes de las elecciones presidenciales de EE. UU, aplaude la visita del candidato demócrata Jesse Jackson a Cuba, y su apuesta por mejorar las relaciones entre ambos países.

A pesar de que estos acercamientos políticos son de gran relevancia, será el factor económico el más llamativo. En el discurso del XXV aniversario del triunfo de la Revolución Cubana en Santiago, Fidel Castro afirma que la economía de la isla creció, pero acepta que más de $80 \%$ del comercio de Cuba depende de países de la órbita soviética o de gobiernos socialistas. Además, sostiene que Cuba creció 2,5 \% en 1982, y 5 \% en 1983. También proyecta para 1984 un crecimiento similar al del año anterior.

Sin embargo, en el discurso de conclusiones del VII periodo ordinario de sesiones de la Asamblea Nacional del Poder Popular, Castro exige una mayor disciplina en el Estado, y muestra un plan económico de ahorro llamado Plan de $1985^{[13]}$. Admite: "Puede ser que haya cosas que las queramos ahora mismo y no podemos hacerlas, pero puede ser que dentro de 10 años y 15 años podamos hacer muchas más obras” (Castro, 1984). De igual forma, indica que la economía creció 7,4 \% en 1984, y que crecerá $5 \%$ en 1985. Por último, acepta que para el próximo año tendrán menos combustible y menos electricidad que en 1984. Esto evidencia cierto desgaste respecto a la planificación de la economía, especialmente si se compara el discurso al inicio de ese año.

Para el año de 1985, el mundo está marcado por una serie de cambios transcendentales, que marcaron un antes y un después para la década de 1980. Ese año fallece el soviético Konstantín Chernenko, el último de una generación política que empezó a irrumpir en Rusia luego de la muerte de Stalin. Un miembro joven del buró político soviético, llamado Mijail Gorbachov, asume temporalmente el cargo ejecutivo, y posteriormente es 
elegido para dirigir la Unión Soviética. En esta nueva etapa, la primera acción que muestra un cambio dentro de la URSS se da con el anuncio de la suspensión del despliegue de misiles de alcance medio en Europa y la renovación de los altos cargos, incluyendo al canciller Andréi Gromyko ${ }^{[14]}$, cuya trayectoria en el cargo era de 28 años. Gorbachov también admite que la economía soviética estaba en estancamiento y propone una serie de reformas llamadas uskoréniye. Para noviembre de 1985, Ronald Reagan y Mijaíl Gorbachov se reúnen por primera vez en Suiza, mostrando un interés mutuo de acercamiento y distención, que no se había evidenciado en años anteriores dentro de ambas naciones.

En la Cuba de 1985, los cambios también se hacen sentir con fuerza. El 1 de enero de ese año, el X Pleno del Comité Central del Partido Comunista Cubano del 1 de enero de 1985 convoca oficialmente el inicio para la realización del III congreso del partido, que marcará la agenda del país durante todo ese año.

No obstante, las acciones a nivel internacional continuaban con la habitual ejecución de eventos internacionales, y con un marcado interés de imponerse en la agenda pública de Latinoamérica. Durante ese año se realizaron el Encuentro sobre la Situación de la Mujer en América Latina y el Caribe, el Encuentro sobre la Deuda Externa de América Latina y el Caribe, el Dialogo Juvenil y Estudiantil de América Latina y el Caribe sobre la Deuda Externa, la Conferencia Sindical de Los Trabajadores de América Latina y el Caribe sobre la Deuda Externa, el IV Congreso de la Federación Latinoamericana de Periodistas, y el VII Festival Internacional del Nuevo Cine Latinoamericano. Se puede apreciar un claro interés por poner el tema de la deuda externa como eje central del discurso político internacional de Cuba. Esto refleja un cambio en comparación a años anteriores, donde se podía evidenciar una mayor diversidad en la agenda pública e internacional del gobierno cubano.

Este cambio de discurso también se puede percibir en declaraciones públicas. En el acto central por el XXV aniversario de la creación de los Comités de Defensa de la Revolución, Fidel Castro reafirma, con más fuerza que años anteriores, que Cuba atraviesa una situación económica deficiente, indica: "En medio de una gran crisis económica, esa es nuestra situación; no es de abundancia, no es de despilfarro, nunca debe ser de despilfarro. No es fácil” (Castro, 1985). De igual forma, señala más adelante durante esa alocución: "Sabemos que tenemos fallas, tenemos defectos y tenemos que luchar incansablemente y sin tregua para vencerlos. Algunos de ellos son más difíciles que otros de aquellos males de que hablamos, y no vamos a hacer el recuento" (Castro, 1985).

Estas declaraciones marcan el inicio de una etapa de reconocimiento sobre ciertos aspectos escamosos que experimenta la gestión del gobierno cubano, con el telón de fondo de un panorama internacional, que empieza a condicionar la posición geopolítica de la isla caribeña. Sin embargo, será con la realización del III Congreso del Partido Comunista Cubano en 1986, que esto estos reconocimientos y cambios resultarán más evidentes.

\section{El tercer congreso}

El III Congreso del Partido Comunista Cubano inicia el 5 de febrero de 1986, con las palabras de apertura del jefe de las Fuerzas Armadas Revolucionarias de Cuba, Raúl Castro. En sus primeras declaraciones, se indica que están presentes más de 186 delegaciones extranjeras para observar el proceso, y 1790 delegados nacionales elegidos para participar en el congreso. Ellos representan a más 40 mil organizaciones que agrupan a más de 1 millón de comunistas cubanos.

Castro establece que el congreso discutirá durante los próximos meses de 1986, el proyecto del primer programa del partido comunista de Cuba, para que pueda ser analizado por otros actores sociales importantes como la Unión de Jóvenes Comunistas, las Fuerzas Armadas Revolucionarias y el sector público en general. La importancia transcendental de este congreso recae en que se definirán los lineamientos políticos, económicos y sociales, que Cuba establecerá por los próximos 5 años. 
Este proyecto del partido comunista cubano y los cambios sugeridos para la dirección política se han disgregado en tres documentos: 1) Resoluciones sobre el perfeccionamiento de la división políticoadministrativa, 2) Resoluciones sobre el perfeccionamiento del sistema de dirección y planificación de la economía, y 3) Resoluciones sobre la política internacional.

Respecto a las Resoluciones sobre el perfeccionamiento de la división político-administrativa, se indica que a pesar de los avances implementados a través del I y II Congreso del PCC, existen municipios con poco desarrollo económico y baja autonomía para resolver problemáticas locales, y que no está bien establecido el estatus institucional de la ciudad de La Habana. En función de estas premisas, en el documento los delegados señalan la necesidad de fusionar o unificar municipios con poca capacidad económica y la aplicación experimental de unidades políticas administrativas de distritos para las grandes ciudades como Santiago de Cuba, Camagüey y La Habana.

Se puede apreciar que más allá del interés político de esta división territorial, la principal preocupación o interés del III Congreso era la autonomía económica. Buscaban organizar el país bajo criterios de dependencia, en función de reducir gastos respecto al gobierno central.

En el caso del documento de Resoluciones sobre el perfeccionamiento del sistema de dirección y planificación de la economía (Partido Comunista de Cuba, 1986a), se puede apreciar una reflexión amplia y profunda sobre la situación de la economía cubana. Se señala el déficit en la balanza de pagos, especialmente en el área de la moneda libremente convertible, el deterioro de los precios del azúcar, la reducción de las fuentes de financiamiento del Banco Nacional de Cuba, el incumplimiento en los plazos para concluir obras, y las deficiencias en el Sistema de Dirección y Planificación de la Economía (SDPE). Ante este contexto, los delegados sostienen la necesidad de elevar la capacidad adquisitiva en el país, la sustitución de las importaciones y un mayor ahorro energético, así como la continuación de una política de austeridad.

A pesar de estos reconocimientos nunca antes hechos hasta ese momento, la intención de la planificación de la economía bajo una lógica socialista no sufre grandes cambios en el marco ideológico. Esto se evidencia en otras sugerencias emitidas por los delgados en el documento. Por ejemplo, se habla sobre la reforma y establecimiento de los precios mayoristas para la industria extractiva y de pesca, y el control sobre los salarios y todas las fases del proceso productivo.

De igual forma, se puede notar que este documento resulta el más extenso realizado durante las resoluciones del III congreso. Esto evidencia que la economía era un tema central dentro de la política cubana y la principal preocupación en el marco de este evento, así como ya se venía reflejando en los discursos de Fidel Castro durante 1984 y 1985.

Respecto al documento de Resoluciones sobre la política internacional, se establece desde su inicio que el objetivo de la política internacional cubana es contribuir al fortalecimiento del socialismo en el mundo, las causas de liberación, el progreso de los pueblos y la paz. De igual forma, señala que la línea política que se sigue está enmarcada en el marxismo-leninismo, el internacionalismo proletariado, y la estrecha colaboración con la Unión Soviética y los países de la órbita socialista, así como los vínculos con el movimiento comunista y los movimientos revolucionarios.

En primera instancia, en este documento también se saluda las gestiones del líder soviético Mijaíl Gorbachov, sobre un programa para la eliminación de armas nucleares y químicas en un plazo de 15 años, así como la no militarización del espacio exterior. Igualmente, los delegados destacan el interés mostrado en el Consejo de Ayuda Mutua Económica (Came), con el fin de profundizar la integración económica de los países socialistas

No obstante, en la geopolítica planificada de Cuba, la región de Latinoamérica es un factor de primer orden dentro de sus intereses. Esto se demuestra en uno de los primeros enunciados de esta resolución, que establece (Partido Comunista de Cuba, 1986b): 
El III Congreso confirma los vínculos indisolubles de la revolución cubana con los pueblos hermanos de América Latina y el Caribe, a los cuales está unida en la historia, en la cultura y en la batalla contra el enemigo común para alcanzar la verdadera y definitiva independencia. (p. 1)

Otro elemento central dentro de la política internacional que se manifiesta en la resolución es la posición cubana frente a los Estados Unidos. En función del contexto por la administración de Ronald Reagan, los delegados comentan (Partido Comunista de Cuba, 1986b):

La aplicación por parte de la administración estadounidense de una política exterior fascista basada en las concepciones chovinistas y militaristas y en el estímulo abierto a la contrarrevolución en todo el mundo provocó una peligrosa agudización de las tenciones, el recrudecimiento de la carrera armamentista y de las medidas de tipo militar, a la vez que llevó en la práctica a la cancelación del proceso de distención internacional. (p. 3)

A pesar de que existía un proceso de distención iniciado en 1985 entre EE. UU. y la URSS, los delegados cubanos niegan esta realidad en 1986, contradiciendo inclusive las posiciones oficiales del gobierno soviético sobre este tema.

También, de forma particular dentro de este documento, se solidarizan con la Revolución Sandinista de Nicaragua, y con el Frente Farabundo Martí para la Liberación Nacional y el Frente Democrático Revolucionario, ambos de El Salvador. Abogan por una solución negociada al conflicto centroamericano a través del apoyo al Grupo Contadora. Esto es un cambio transcendental en la política exterior cubana hacia Centroamérica, ya que desde la década de 1970 la línea política se basaba en el apoyo explícito a la lucha armada por parte de grupos comunistas para alcanzar el poder.

Se menciona la solidaridad con la oposición chilena, la causa independentista de Puerto Rico, y el apoyo hacia Argentina por el conflicto de las Islas Malvinas, así como los procesos de apertura de democrática que se gestan en Uruguay y Brasil.

Respecto a otras regiones del mundo, los delegados manifiestan su apoyo hacia el Congreso Nacional Africano y rechazo al apartheid en Suráfrica. También, se manifiesta la solidaridad con el gobierno de Angola, la lucha guerrillera de la Organización del pueblo de África del Sudoeste (Swapo) en Namibia, y el apoyo a la Revolución Etíope, así como a las naciones de Sudan, Ghana, Burkina Faso, la República Árabe Saharaui Democrática, Palestina y el Líbano. Por último, indican la importancia estratégica que representa el Movimiento de Países No Alineados para la política internacional cubana, en el marco de procesos de pacificación.

Las posturas expuestas en estas resoluciones permiten un primer acercamiento al planteamiento político de la Cuba de Fidel Castro para 1986. Se pueden observar las génesis de procesos de cambio, principalmente a nivel económico, a través del autoreconocimiento de las fallas. Esto fue algo impensable en un primer momento, sobre todo por el comportamiento autoritario del gobierno cubano frente a las críticas.

Por ese hecho transcendental, en el marco del III congreso, los comunistas cubanos bautizaron a esta serie de cambios bajo el nombre de "Proceso de Rectificación de Errores y Tendencias Negativas". Según el investigador social Carmelo Mesa-Lago ${ }^{[15]}$, con este proceso se pretendió implementar un nuevo modelo con mayor centralización económica, ya que las gestiones del Sistema de Dirección y Planificación de la Economía, entre 1976 y 1985, se traducían en pérdida del control real del buró político comunista frente a la economía del país. Este autor señala que, para la fecha, este tipo de actitud resultaba contraria a las reformas económicas iniciadas en la Unión Soviética y en China.

No obstante, este hecho de rectificación dentro del contexto histórico de 1986 y la dinámica del III congreso no se puede ubicar únicamente en los registros publicados por el Partido Comunista Cubano. Resulta necesario conocer los discursos y acciones políticas por parte de Fidel y Raúl Castro durante ese año, ya que también pueden sugerir otros elementos adicionales en este proceso de reconocimiento y adaptación.

El investigador en filosofía Jesús García Brigos (2004) indica que se puede tomar como punto de partida la "Rectificación de Errores y Tendencias Negativas" en el discurso de Fidel Castro del 19 de abril de 1986, en el 
marco del XXV aniversario de la victoria de la Batalla de Girón. Al analizar la alocución sugerida por García Brigos, se pueden hallar ciertas afirmaciones que sugieren un proceso de cambio en el aspecto económico (Castro, 1986a):

En nuestro país actualmente se discute el Programa del Partido entre todo el pueblo. A fines de año, en una reunión especial, los delegados al Tercer Congreso analizarán y aprobarán ese Programa. Las condiciones en que ese gran Programa tendrá que cumplirse no son fáciles; incluso, las condiciones en que tenemos que cumplir nuestros planes económicos: el plan del año 1986, el plan del quinquenio y los planes perspectivos no son nada fáciles. Un día como hoy también tenemos que meditar en estas cosas. Hay circunstancias, algunas surgidas incluso después del Congreso, que hacen más difícil la tarea, aunque no irrealizable.

En el fragmento anterior se puede apreciar el reconocimiento por parte de Castro del inicio de un nuevo proceso económico, e indica que no será fácil de asumir. De igual forma, más adelante también señala (Castro, 1986a):

En el Congreso del Partido se trazaron líneas muy precisas. Se hicieron críticas duras, agudas, a los problemas que subsisten; y nos hicimos el compromiso de luchar contra esos problemas, detectarlos uno por uno y combatirlos. Estamos discutiendo el Programa, como decíamos anteriormente. Sin estos problemas a los que me refiero, habría que cumplir esa línea trazada por el Congreso, pero hoy con las dificultades mayores que tendremos es mucho más importante y más decisivo cumplir esa línea. Tenemos que ser mucho más intransigentes contra todo lo mal hecho, contra todo lo incorrecto; tenemos que llevar una lucha mucho más consecuente contra problemas que subsisten, o nuevos problemas que se desarrollan.

A pesar de que no se señala explícitamente las dificultades que atraviesa Cuba, sí existe un reconocimiento claro de una situación compleja, que requiere medidas para solventarla. Si se considera el habitual estilo de Castro para enmarcar los logros del gobierno cubano bajo una perspectiva optimista y reivindicativa, estas palabras sugieren un cambio importante respecto a su postura pública.

Durante este discurso, Fidel Castro también señala cuáles son los factores que desea cambiar dentro de este proceso económico, bajo un prisma comunista y antagonista del sistema capitalista de Occidente (Castro, 1986a):

Nuestro Partido tiene que estar alerta, ¡muy alerta! Tenemos medio millón de militantes del Partido, entre militantes y aspirantes, una fuerza tremenda — isi nosotros, cuando empezamos la lucha contra la tiranía, hubiéramos tenido una organización de medio millón de militantes!-; más otro medio millón de militantes en la juventud, qué fuerza increíble; las organizaciones de masa, los millones de hombres y mujeres organizados en los sindicatos, los comités de defensa, la federación, los pioneros, ¡una fuerza tremenda, increíble!, con ella realmente bien utilizada podemos ir poniéndole fin a todas esas cosas sin extremismos; no tenemos que caer en ningún extremismo, ni en campañas de unos meses, ¡no!, esta tiene que ser una lucha sostenida. Como les decía a los pioneros, hay que seguir luchando contra la reminiscencia del viejo sistema, contra la reminiscencia del capitalismo, de la ideología del capitalismo, contra el parasitismo, contra los privilegios, contra esa tendencia a recibir bienes sin proporción al trabajo que se aporta a la sociedad. Hemos hablado de la fórmula socialista: ¡De cada cual, según su capacidad, a cada cual según su trabajo!; es bien clara esa fórmula, no es, desde luego, igualitarista.

En otros discursos de Fidel Castro en 1986, se puede observar que esta actitud de reconocimiento e impulso de nuevas medidas económicas resulta una constante, además de admitir la existencia de corrupción en el sistema estatal. En el discurso emitido el 18 de mayo, en el marco del II Encuentro Nacional de Cooperativas de Producción Agropecuaria, indica (Castro, 1986b):

En el esfuerzo por buscar la eficiencia económica, hemos creado el caldo de cultivo de un montón de vicios y deformaciones, y lo que es peor, ¡corrupciones! Eso es lo que duele. Todo eso puede mellar el filo revolucionario del pueblo, de nuestros trabajadores, de nuestros campesinos. Y eso sí es muy malo, porque debilita a la Revolución, no solo políticamente, incluso militarmente la debilita; porque si nosotros tenemos una clase obrera que se deja llevar nada más por el dinero, que empieza a ser envilecida por el dinero, que no actúa más que por el dinero, entonces estamos mal, porque de ese tipo de hombre no sale un defensor óptimo de la Revolución y de la patria.

Cosas nuevas se aprenden constantemente en el proceso revolucionario. Así, la rectificación de determinados errores ha creado otros errores, la rectificación de determinados vicios ha creado otros vicios. Pero — como decía- la Revolución tiene ya suficiente experiencia para saber rectificar esos errores sin caer en otros, para saber rectificar esos vicios sin caer en otros. Y 
en esta gran cruzada, en esta gran lucha por el saneamiento contra todos esos vicios, contra todas esas manifestaciones, nos complace mucho saber que contamos con el apoyo decidido y entusiasta de nuestros campesinos cooperativistas.

Bajo la misma línea discursiva, en el III Congreso de los Comités de Defensa de la Revolución, el 28 de septiembre de 1986, Fidel Castro afirma la existencia de la especulación, la necesidad de perfeccionar la economía para salvar la estabilidad del gobierno y la resistencia que puede existir en algunos sectores de la sociedad cubana, que se benefician de la especulación (Castro, 1986c).

Estas declaraciones de Fidel Castro en diferentes momentos de 1986 demuestran el interés en torno a un proceso de rectificación en el seno del III Congreso del PCC, que transcienden las resoluciones expuestas anteriormente. Eso se puede observar, también, en el discurso de apertura de Raúl Castro el 30 de noviembre de 1986, cuando el congreso vuelve a reunirse para fijar su posición final, luego de meses de un proceso de consulta.

Raúl Castro (1986) sostiene en su discurso que con este congreso se da inicio a una etapa superior en la historia de la Revolución Cubana. Señala la importancia de Fidel Castro, quién gestionó durante varios meses el análisis sobre los errores y desviaciones en la dirección económica y política, así como la necesidad de rectificación. También en esta alocución reafirma que la razón de diferir las sesiones del III Congreso obedecieron a llevar por la vía del hecho la aprobación del primer programa del PCC con los militantes y trabajadores de todo el país.

Por su parte, Fidel Castro, en el discurso de clausura del III Congreso del Partido Comunista Cubano el 2 de diciembre de 1986, menciona que las decisiones tomadas a partir de las resoluciones y tesis expuestas deben sustituir al Programa Moncada instaurado al inicio de la Revolución Cubana como plan de gobierno.

Entre las primeras palabras que destaca Castro en este discurso, se puede apreciar el reconocimiento de errores desde la perspectiva del PPC (Castro, 1986d):

Nos lo estaban empezando a echar a perder; pero muy a tiempo hemos reaccionado para que no nos corrompan a los militantes, para que no nos corrompan al Partido, para que no nos corrompan al pueblo, para que no nos corrompan a los jóvenes y, sobre todo, para que no corrompan a nuestra clase obrera. No estoy hablando para expresar una ilusión, sino para expresar lo que hemos estado viendo en este proceso de rectificación.

Luego de este planteamiento, se puede incluso sugerir la existencia de un enemigo interno dentro de su propio partido, por las divagaciones al momento de señalar culpables. Castro también señala, desde una visión histórica, la mala interpretación política de ciertos mecanismos implementados por el gobierno y sus consecuencias para aquel momento, bajo el contexto de la rectificación de errores (Castro, 1986d):

Aquí hemos incurrido en dos tipos de ilusiones. Cuando se promulgó la Constitución, se llevó a cabo la división política administrativa y se desarrollaron los Poderes Populares, que fue un gran avance, sin duda, se originó la creencia ingenua de que a partir de esas innovaciones y de esos avances el Estado iba a funcionar de una manera perfecta, casi automáticamente. Después se fue viendo que esto requería un trabajo político muy importante, un trabajo inmenso del Partido. Por otro lado, en la esfera de la producción material y de los servicios, especialmente en la esfera de la producción material, se llegó a la creencia de que todo marcharía a la perfección con el Sistema de Dirección y Planificación de la Economía, con la vinculación del salario y el trabajo, la panacea que lo resolvería todo y, casi, casi iba a construir el socialismo.

Ahora estamos enfrascados en este proceso de rectificación, de lucha contra las tendencias negativas —como les explicaba a ustedes-, en medio de una situación económica peculiar. No digo que es difícil, porque difícil puede dar la idea de que es difícil en todo. Por eso digo peculiar, porque hay algunos aspectos de la economía que tienen situaciones complicadas, difíciles, no todos. No le va a faltar al país, por ejemplo, el combustible que necesita, lo tiene asegurado; no le van a faltar muchas cosas que tiene aseguradas en sus relaciones económicas con los países socialistas. Pero sí nos van a faltar cosas de las que tenemos que importar del área de divisas convertibles; ¡sí nos van a faltar, y sí tenemos en eso una situación complicada, que nos va a traer problemas! Esa situación que tenemos puede traernos problemas de atrasos en llegadas de materias primas, puede traernos dificultades en piezas, pueden faltarnos algunas, pueden llegar tarde otras, debido a las limitaciones que tenemos con las divisas convertibles, grandes, ¡más grandes que nunca!

En este fragmento se puede notar cómo Fidel Castro anticipa la crisis económica de Cuba, que alcanzaría su punto máximo en la década de 1990. En un momento donde todavía existía la Unión Soviética y la cortina 
de hierro, que Castro admita las carencias económicas que sufrirán próximamente demuestra que la economía cubana y su estancamiento no tuvieron relación con la desaparición de la URSS, sino que sucedieron mucho antes y a nivel interno.

Por último, Castro atribuye la precariedad del contexto de limitaciones económicas a la deuda externa y los fenómenos naturales de 1985 y 1986, que limitaban la capacidad financiera de la isla (Castro, 1986d):

Yo trataba de explicar a los compañeros en el Congreso - creo que algo de eso salió por la prensa- y decía que íbamos a disponer, para las importaciones en convertible, de la mitad de lo que tradicionalmente hemos dispuesto, y unas importaciones que en el pasado eran como mínimo de 1200 millones de dólares se verán reducidas a 600 millones. Y tenemos que arreglárnoslas con esas cantidades de dólares y debemos estar preparados para esas dificultades, que se van a presentar, desgraciadamente, porque a veces usted no puede comprar hasta que no dispone del dinero; y no puede gastar a menos que ingrese. En esto está implicado también la situación de la deuda externa y los problemas que trae aparejados, de los cuales se ha hablado bastante y que están padeciendo muchos países; situación — como he explicado otras veces-agravada para nosotros este año por una serie de factores ya de orden objetivo, como fue la sequía del año pasado, más el ciclón. Pero el año pasado el esfuerzo que se hizo logró reducir el daño del ciclón en la producción azucarera a mucho menos de lo que había producido la sequía.

Ante esta realidad de Cuba, no resulta difícil entender por qué en el plano internacional, durante 1985 y 1986, se invirtió dinero y esfuerzo para denunciar la crisis de la deuda externa en los países del tercer mundo, ya que también era un forma de consolidar un respaldo internacional, que permitiera obtener más tiempo y recursos para el pago de la deuda.

Bajo esa misma lógica de mantener los escenarios internacionales abiertos, mientras el Partido Comunista de Cuba discutía y sometía a consulta los cambios políticos internos necesarios para mantener la estabilidad del gobierno, la agenda internacional de Fidel Castro continuaba. Ese año fue invitado al XXVII Congreso del Partido Comunista de la Unión Soviética, y participó en la Cumbre del Movimiento de Países No Alineados en Zimbabue. Todas estas acciones se puede inferir como una búsqueda de Cuba de fortalecer las relaciones diplomáticas y económicas con países con afinidad ideológica. En marzo de 1986, Castro visitó la República Popular Democrática de Corea y señaló (Castro, 1986e):

Llegamos a Corea movidos por un profundo interés político; deseamos también apreciar su original y milenaria cultura, y aspiramos a conocer al máximo las admirables realizaciones de este pueblo y sus experiencias revolucionarias. Aunque el tiempo de que dispondremos, por razones ajenas a nuestra voluntad, no será todo el que hubiésemos deseado, estamos seguros de que nuestros contactos, nuestras conversaciones con el querido presidente Kim Il Sung y con nuestros hermanos coreanos, darán un nuevo impulso a nuestra amistad, a nuestro conocimiento recíproco, a la cooperación y a la solidaridad entre ambos partidos, estados y pueblos.

Al finalizar la visita a Corea del Norte, Fidel Castro en su discurso de despedida señala y agradece de forma explícita al gobierno norcoreano (Castro, 1986f):

Aprovecho esta oportunidad, queridos hermanos coreanos, para agradecer al compañero Kim Il Sung y al gobierno de la República Popular Democrática de Corea, por habernos suministrado, mediante créditos altamente favorables y a precios de costo en meses recientes, 100.000 fusiles y decenas de millones de balas como contribución, junto a la URSS y otros países socialistas, al esfuerzo de nuestro pueblo para fortalecer las defensas de nuestra patria también amenazada por los imperialistas yanquis.

Estas maniobras diplomáticas de 1986 sugieren la necesidad de Cuba de mantener el vínculo con la Unión Soviética a pesar de los cambios internos impulsado por Gorbachov. De igual forma, sugieren la necesidad de buscar mayor diversidad en el intercambio comercial, siempre bajo un enfoque estratégico. En este caso, al analizar la visita de Castro a Corea del Norte, resulta evidente que no solo existe un interés comercial, sino también militar. 


\section{Antesala a un viraje}

Las decisiones tomadas por el Partido Comunista de Cuba, al considerar los años previos a 1986, se pueden entender como una reacción ante un esquema geopolítico que ya no era del todo favorable, y empezaba a afectar con mayor fuerza su economía. El envejecimiento de la cúpula soviética y los cambios seguidos de su liderazgo, la ofensiva militar y política de Estados Unidos en América Latina y el Caribe, y la mala planificación económica y la recesión se puede pueden considerar como los factores de mayor influencia que llevaron a la Revolución Cubana a este proceso de "rectificación" y, hasta cierto punto, de anticipación del proceso de periodo especial, que atravesará la isla caribeña en la década de 1990.

Por otra parte, si bien es cierto que ante los argumentos y discursos expuestos en el marco del III Congreso del Partido Comunista Cubano y los discursos de Fidel y Raúl Castro, no se evidencian grandes cambios en un primer momento, las decisiones tomadas durante ese año serán la base fundamental para un cambio en la política de forma gradual, anticipando un escenario donde ya Cuba no gozaría de la misma influencia, y las relaciones económicas con los países socialistas se deteriorarían.

Dentro de una perspectiva histórica se puede cuestionar qué tan exitosa fue esa anticipación estimada, sobre todo a nivel económico. Sin embargo, a nivel político, especialmente en la geopolítica, se encuentran signos que pueden sugerir cambios importantes:

1) Una actitud más abierta hacia la Iglesia Católica al permitir el Encuentro Nacional Eclesiástico de Cuba en 1986, esto se puede entender como un primer acercamiento hacia el Vaticano, especialmente hacia Juan Pablo II.

2) El apoyo hacia la firma del tratado de eliminación de armas nucleares por parte de la EE. UU y URSS en 1987.

3) El saludo hacia el acuerdo Esquipulas I y II para Centroamérica. Si se considera la actitud de años anteriores a nivel de confrontación, bajo el esquema de la Guerra Fría y el apoyo mostrado a movimientos subversivos centroamericanos, este es un cambio significativo.

4) El apoyo explícito a la Unión Patriótica de Colombia para alcanzar el poder por vías electorales en 1986.

5) La colaboración de forma directa para crear, en 1990, el grupo "Foro de São Paulo", en Brasil.

A nivel interno, para el año de 1987, a pesar de iniciar grandes cambios económicos, el PIB de Cuba se encuentra en -2,402\%. Esto demuestra el porqué de la preocupación de Castro y del partido por hablar sobre la economía y la necesidad de cambio en las políticas de la isla caribeña.

El próximo congreso del Partido Comunista de Cuba sería 6 años más tarde, en 1991, bajo un contexto mundial muy diferente y complicado, marcado por la caída del Muro de Berlín (1989), la invasión de Estados Unidos a Panamá (1989), la separación del poder del FSLN de Nicaragua (1990), la disolución del Came (1991), y la desintegración de la Unión Soviética (1991).

En función de esto, desde el análisis histórico, el III Congreso del PPC de 1986 fue el inicio de un proceso de transición, adaptación y preparación hacia la etapa más compleja vivida por el gobierno cubano en la década de 1990: El periodo "especial" ${ }^{[16]}$. Sin embargo, las acciones del III congreso influyeron notablemente, no solo en la dinámica política de Cuba, sino en la dinámica geopolítica de América Latina, ya que estimuló un periodo de mayor distención entre sus aliados.

A luz del año 2017, y con un Raúl Castro que señala que dejará la presidencia en 2018, no se puede olvidar, la influencia de Cuba en ciertos países, como es el caso del gobierno de Venezuela o Colombia a través del partido político Fuerza Alternativa Revolucionaria del Común. Resulta necesario conocer la historia del Partido Comunista Cubano, ya que, gracias a su visón expansionista y la transcendencia de los planteamientos esgrimidos en su seno, se pueden identificar ciertas directrices que toman las izquierdas latinoamericanas y sus gobiernos. 


\section{Referencias}

Banco Mundial. (s. f.). Datos de Cuba. Crecimiento del PIB (\% anual). Recuperado de https://datos.bancomundial .org/pais/cuba

Carter, J. (1980, 7 de enero). Shipments of Agricultural Commodities to the Soviet Union, Memorandums from the President. Recuperado de https://www.presidency.ucsb.edu/node/250453

Castro, F. (1982). Clausura de la Reunión de la Presidencia del Consejo Mundial de la Paz. Recuperado de https://w ww.cuba.cu/gobierno/discursos/1981/esp/f210481e.html

Castro, F. (1983a). Acto central por el XXX Aniversario del Ataque al Cuartel Moncada. Recuperado de https://ww w.cuba.cu/gobierno/discursos/1983/esp/f260783e.html

Castro, F. (1983b). Acto de despedida de duelo a los héroes caídos en desigual combate frente al Imperialismo Yanki en Granada. Recuperado de https://www.cuba.cu/gobierno/discursos/1983/esp/f141183e.html

Castro, F. (1984). Conclusiones del VII Periodo Ordinario de Sesiones de la Asamblea Nacional del Poder Popular. Recuperado de https://www.cuba.cu/gobierno/discursos/1984/esp/f281284e.html

Castro, F. (1985). Acto Central por el XXV Aniversario de la creación de los Comités de Defensa de la Revolución. Recuperado de https://www.cuba.cu/gobierno/discursos/1985/esp/f280985e.html

Castro, F. (1986a). Acto central por el XXV Aniversario de la Victoria de Girón. Recuperado de https://www.cuba. cu/gobierno/discursos/1986/esp/f190486e.html

Castro, F. (1986b). Conclusiones en el II Encuentro Nacional de Cooperativas de Producción Agropecuaria. Recuperado de https://www.cuba.cu/gobierno/discursos/1986/esp/f180586e.html

Castro, F. (1986c). Conclusiones en el III Congreso de los Comités de Defensa de la Revolución. Recuperado de htt ps://www.cuba.cu/gobierno/discursos/1986/esp/f280986e.html

Castro, F. (1986d). Clausura de la Sesión diferida del Tercer Congreso del Partido Comunista de Cuba. Recuperado de https://www.cuba.cu/gobierno/discursos/1986/esp/f021286e.html

Castro, F. (1986e). Banquete Ofrecido en Honor de la Delegación del Partido y el Gobierno de la República de Cuba, por el Mariscal Kim Il Sung, Secretario General del Partido del Trabajo y Presidente de la República Popular Democrática de Corea. Recuperado de https://www.cuba.cu/gobierno/discursos/1986/esp/f080386e.html

Castro, F. (1986f). Acto de masas celebrado en el Palacio de los Deportes de la Ciudad de Pyongyang. Recuperado de https://www.cuba.cu/gobierno/discursos/1986/esp/f110386e.html

Castro, R. (1986). Palabras pronunciadas por Raúl en la apertura del III Congreso. Recuperado de https://www.granma.cu/file/pdf/PCC/3congreso/Palabras-pronunciadas-por-Ra\%C3\%BAl-en-la-apertur a-del-III-Congreso1.pdf

García Brigos, J. (2004). Proceso de Rectificación y salida del Periodo Especial: dos fases en la misma batalla de ideas. La Habana: Cuba Siglo XXI. Recuperado de https://www.nodo50.org/cubasigloXXI/politica/gbrigos_proc_ rectif.pdf

Partido Comunista de Cuba. (1986a). Tercer Congreso del Partido Comunista de Cuba. Resoluciones sobre el perfeccionamiento de la división política-administrativa del país. La Habana: Editora Política. Recuperado de https://www.granma.cu/file/pdf/PCC/3congreso/Tesis\%20y\%20resoluciones/III-Congreso-PCC.-Resolu ciones-sobre-el-perfeccionamiento-de-la-divisi\%C3\%B3n-pol\%C3\%ADtico-administrativa.pdf

Partido Comunista de Cuba. (1986b). Tercer Congreso del Partido Comunista Cubano. Resoluciones sobre la política internacional. La Habana: Editora Política. Recuperado de https://www.granma.cu/file/pdf/PCC/3congreso/ Tesis\%20y\%20resoluciones/III-Congreso-PCC.-Resoluciones-sobre-la-pol\%C3\%ADtica-internacional.pdf 


\section{Notas}

[1] El éxodo del Mariel se considera uno de los más grandes movimientos migratorios del siglo XX, ya que en siete meses (entre abril y octubre de 1980) 125 mil cubanos salieron de la isla caribeña con destino a Estados Unidos, especialmente a la ciudad de Miami.

[2] Frente Farabundo Martí para la Liberación Nacional. Se trata es un movimiento guerrillero y posterior partido político de centroizquierda/izquierda de Guatemala, creado en 1980.

[3] Ejército Guerrillero de los Pobres de Guatemala. Creado en 1972, conformó la organización Unidad Revolucionaria Nacional Guatemalteca.

[4] Guerra de las Malvinas. Se trata de un enfrentamiento bélico entre Argentina y el Reino Unido, en 1982. El origen del conflicto fue el intento por parte de Argentina de recuperar la soberanía de las islas Malvinas, ubicadas al este de su territorio. Según las Naciones Unidas, las islas se consideran territorios en litigio entre Argentina y el Reino Unido. Esta disputa estimuló una unidad coyuntural de toda América Latina hacia Argentina, a pesar de las diferencias ideológicas.

[5] El Movimiento de Países No Alineados es una agrupación de naciones creada en 1961, cuyo objetivo principal fue establecer una posición de autonomía frente a las potencias de los Estados Unidos y la Unión Soviética, en el marco de la Guerra de la Fría.

[6] Efraín Ríos Montt. Fue un militar de Guatemala, que ejerció una dictadura en el país centroamericano, entre 1982 y 1983. Actualmente es acusado de genocidio por la justicia internacional.

[7] Sistema Estratégico de Defensa. Se trató de una iniciativa propuesta por el Presidente de los Estados Unidos, Ronald Reagan, en 1983. El objetivo era utilizar sistemas basados en tierra y en el espacio con el fin de defender a los Estados Unidos contra un ataque nuclear con misiles balísticos intercontinentales y misiles balísticos lanzados desde submarinos.

[8] Fundación Nacional para la Democracia. Se trata de una organización creada en 1983 y financiada por el Congreso de los Estados Unidos. El objetivo de esta institución es apoyar a diversos organismos y personas que trabajen a favor de la democracia en América Latina.

[9] Invasión de Estados Unidos a la isla de Granada. Se trató de una operación militar de Estados Unidos en la isla caribeña de Granada, cuyo objetivo era derrocar la implementación de un gobierno comunista prosoviético.

[10] En 1983, la región de América Latina y el Caribe atravesó convulsiones de inestabilidad política por la operación militar de Estados Unidos hacia la isla de Granada, con el objetivo de deponer un gobierno, ideológicamente relacionado con la Unión Soviética y el gobierno cubano.

[11] Grupo Contadora. Se trató de una alianza de México, Colombia, Panamá y Venezuela, creada en enero de 1983. El objetivo era promover conjuntamente la paz en las naciones Centroamérica, asediadas por las guerras civiles y gobiernos autoritarios.

[12] Central de Trabajadores de Cuba.

[13] Se trató de un plan estratégico, cuyo fin era establecer metas de producción y gastos públicos para obtener mayores ahorros.

[14] Andréi Gromyko. Fue el canciller de la Unión Soviética entre 1957 y 1983. Se considera como una de las figuras internacionales más importantes durante la Guerra Fría. Protagonizó la Crisis de los misiles en Cuba, así como la promoción de la distención entre las superpotencias.

[15] Investigador titular del Instituto de Filosofía del Ministerio de Ciencia, Tecnología y Medio Ambiente de la Habana, que trabaja temas relacionados con el materialismo histórico y la sociedad cubana, desde una visión integral.

[16] Período Especial. Se trató de una depresión económica de la economía cubana, en la década de 1990, motivada por la caída de la Unión Soviética, principal proveedor de la isla, así como por un recrudecimiento del embargo impuesto por Estados Unidos.

* Artículo de investigación

\section{Licencia Creative Commons CC BY 4.0}

Cómo citar este artículo: Carrasco Muro, C. D. (2019). El primer punto de cambio del gobierno cubano: III Congreso del Partido Comunista Cubano de 1986. Papel Político, 24(1). https://doi.org/10.11144/Javeria na.papo24-1.ppcg 